\title{
Placental Vasculogenesis and Angiogenesis Related to GDM and the Possible Affect on the Placenta and the Fetus Outcome
}

\author{
RafahHadyLateef ${ }^{1}$, Jinan Mohammed AbdALzahra ${ }^{2}$ \\ 1Ph.D. Student, University of Babylon College of Science for Women, Department of Biology, Iraq, \\ ${ }^{2}$ Assist. Prof., University of Kufa, College of Science, Department of Biology, Iraq.
}

\begin{abstract}
Placentas from gestational diabetes mellitus (GDM) are Patterns of fetoplacental angiogenesis of GDM often hypervascularized. Placenta is a mirror that reflects the well-being of the fetus and continuously undergoes a change in weight, structure, shape and function in order to support the well-being of the fetus.

Aim of Study: In this work we attempted to identify villous vascular and morphological changes in a group of term placentae from mothers with GDM complicating pregnancy.

Objective: To analysis on the possible gross morphological changes and Patterns of fetoplacental angiogenesis of GDM.

Materials and Method: The study was observational, analytical and cross sectional. The patients under this study were selected from the Obstetric of AL-Emam AL-sadiq in the Babylon province. A total of eighty samples were collected from women completed weeks of gestation. Among them, 40 samples were from mothers having GDM, 40 belonged to normal pregnancy (control group). The placentas were examined to measure their weight, diameter, thickness, cotyledons number, Insertion of umbilical cord, Vascular pattern and Fasting blood glucose were determined by enzymatic method using Envoy ${ }^{\circledR} 500$ reagents .Beta chorionic gonadotrophin, progesterone and estradiol were determined using chemilumiscence immunoassay technique on MAGLUMI 600 analyzer.
\end{abstract}

Results: In this study, the GDM group showed significantly higher values for the variables of the weight, thickness,diameter, number of cotyledons, Patterns of fetoplacental angiogenesis of GDM and placentas hypervascularized as compared to normal group. Consequences of GDM include increased risk of macrosomia and birth complications in the infant. Fasting plasma glucose FBG, $\beta-\mathrm{HCG}, \mathrm{P} 4$, E2 were increased in the gestational diabetic women.

Conclusion: From the findings of this study, placental variations in diabetic and normal mothers during pregnancy. The variations in placental weight, thickness, diameter and fetoplacental angiogenesis found in gestational diabetic mother may be a long term compensatory mechanism, aiming to secure a sufficient nutrient supply to support the growth of the foetus. So, postnatal examination of the placenta can yield information that may be important for immediate and late management of the mother and neonate. Fasting plasma glucose FBG, $\beta$-HCG, P4, E2 were increased in GDM group was aimed at assessing placental peptides and maternal factors as potential predictors of the development of gestational diabetes among pregnant women. GDM appears to effect the microvascular remodeling at angiogenesis. So gestational diabetes mellitus (GDM) need to be identified early in pregnancy and managed to maintain a normal vasculature and prevent neonatal mortality and morbidity if rapid intervention is completed.

Keywords: Placenta, GDM, Gross morphology, vasculogenesis, angiogenesis.

\section{Introduction}

The placenta is a transient organ that forms during pregnancy to support the growth and development of the fetus. During placental development, trophoblast cells differentiate through two major pathways. In the villous pathway, cytotrophoblast cells fuse to form 
multinucleated syncytiotrophoblast. In the extravillous pathway, cytotrophoblast cells acquire an invasive phenotype and differentiate into either (first) interstitial extravilloustrophoblasts, which invade the decidua and a portion of the myometrium, or (second) endovascular extravilloustrophoblasts, which remodel the maternal vasculature. These differentiation controlled by the interplay of oxygen tension, transcription factors, hormones, growth factors, and other signaling molecules. Abnormal placental development, the limited invasion of trophoblast cells into the uterus and the following failure of the remodeling of maternal spiral arteries. ${ }^{1}$ The vascularity of placental tissue is dependent on various factors of which fetomaternal hypoxia plays a major role. Hypoxia can be of different types and each type influences the vascularity of the terminal villi. Chorangiosis was the most frequently identified lesion in the GDM group while normal group had normal villous vasculature. Maternal diseases have a major role in disrupting the placental vasculogenesis and angiogenesis by making a hypoxic that may distress the fetus harmfully. The trophoblasts is epithelial cells have functioning as a precursor for the syncytiotrophoblast and extravilloustrophoblasts (EVT). cytotrophoblasts fuse and undergo biochemical differentiation, giving rise to the multinucleated syncytiotrophoblast. Cytotrophoblasts may also acquire invasive properties, forming the EVT. These trophoblasts are able to invade and remodel maternal tissues (interstitial EVT) and uterine spiral artery (endovascular EVT), leading to the widening of artery lumen, reducing the resistance against blood flow that irrigates the fetus. EVT may also invade and remodel uterine glands (endoglandular EVT), which is important to provide nutrition to the embryo. Trophoblast interact with each other and with decidual cells, Hofbaüer cells, endothelial cells, vascular smooth muscle cells, providing a sole microenvironment that is vital for pregnancy outcome and fetal development. ${ }^{2}$ Fetal growth requires remodelling of maternal spiral arteries to provide an adequate maternal blood supply to the placenta. This arterial transformation is achieved by placental trophoblast cells, which invade into the uterine wall. Fetal growth restriction is associated with reduced remodelling of maternal spiral arteries by trophoblast cells. In normal pregnancy, extravilloustrophoblast cells migrate as far as the myometrium and also infiltrate into the arterial media and endothelium of maternal spiral arteries. This results in dilatation and increased flow of maternal blood at low pressure into the intervillous space. In pregnancies affected by gestational complication, the depth of trophoblast invasion is reduced with less spiral artery remodelling. Blood flows at higher pressure and is more pulsatile, resulting in placental stress, reduced placental development and poor fetal growth. Underinvasion is associated with fetal growth restriction; but if invasion is excessive large babies can result. A growing body of evidence is controlled by interactions between killer-cell immunoglobulin-like receptors expressed on maternal uterine natural killer cells (uNK). ${ }^{3}$ Fetal growth in utero depends on the development of a good maternal blood supply to the placenta that requires modification of the uterine spiral arteries. Trophoblast cells from the placenta invade deeply into the stroma to effect arterial conversion. The extravilloustrophoblast cells (EVT) encircle the arteries, and then cause direct destruction of the smooth muscle of the arterial wall with complete loss of vasoconstriction. Trophoblast cells only move down the inside of the arteries to replace the endothelium and functionally modify the media. ${ }^{4}$ The angiogenic process is divided broadly into three major steps including the initiation of the angiogenic response, endothelial cell (EC) migration, proliferation and tube formation, and finally the maturation of the neovasculature. Angiogenesis is a complex, highly regulated process, involving the sprouting, splitting, and remodeling of the existing vessels. ${ }^{5}$

\section{Material and Method}

\section{Inclution Criteria}

\section{Pregnancy women with gestational diabetes} mellitus.

Exlusion Criteria: Pregnant women with a complication, such as gestational hypertension and pregestational DM (Preexisting type I and Type II diabetes mellitus), had a twin pregnancy, and hypertension or other chronic diseases were excluded.

A cross-sectional case-control study from January to March 2019. A total of 80 pregnant women 40 fore each group. The study procedure was approved by the ethics committee of the First Affiliated Hospital Department of AL-Emam AL-sadiq in the Babylon province . Informed agreement was obtained from each participant. Placentas and umbilical cords were collected after delivery from full-term within 36 th to 40 th week of gestation, were included in this study of normal and gestational diabetic groups. Patients and normal pregnant women, who were attended a pretested guided questionnaire. 
The participant females were asked to undergo for Oral Glucose Tolerance Test (OGTT). The control women were within the accepted normal ranges of blood glucose level from 90 to $115 \mathrm{mg} / \mathrm{dL}$ throughout gestation while the GDM women initially showed levels ranging from 120 to $160 \mathrm{mg} / \mathrm{dL}^{6}$

Macroscopic Examinations: Morphological variables of each placenta groups were studied: Immediately after the delivery, within 20 minutes after delivery placentas and umbilical cord were collected after delivery from 40 full-term normal or 40 fullterm gestational diabetic. The placental membranes were clipped and blood coagulants were removed. The placental weight, diameter and thickness were recorded. Diameter of the placenta. The membranes were trimmed from their edges. Blood was removed gently from both surfaces with cotton wool and the umbilical cord was cut about $2 \mathrm{~cm}$ proximal to its insertion. Then the maximum diameter of each placenta was measured with a metallic scale graduated in centimeters, the second maximum diameter was recorded. Then the mean of these two measurements was considered as the diameter of each placenta. Placental thickness measured by piercing a metallic scale through central part including the whole thickness of the placenta were taken from the center of the placenta beside the site of attachment of the umbilical cord. Then separation of the cotyledons to make them prominent in the maternal surface. Counting was started from left side of one end and going through rightward. In this way, counting was continued in spiral manner.

For histological studies, full depth tissue samples. The tissue was cut into $\sim 0.5 \mathrm{~cm} 3$ pieces from placenta on the central and marginal areas. Normal saline was used to wash the pieces before they were dissected into small sections, were placed in $10 \%$ formal-saline for 24-48 hours and were subsequently embedded in paraffin. The $4-\mu \mathrm{m}$ thick sections were stained with Haematoxylin \& Eosin. Numbers and diameter of blood vessels of TV were measured using stage, ocular and reticule micrometers.

Fasting blood glucose were determined by enzymatic method using Envoy® 500 reagents (Vital Diagnostics,
USA). Beta chorionic gonadotrophin, progesterone and estradiol were determined using chemilumiscence immunoassay technique on MAGLUMI 600 analyzer. The sandwich chemilumiscence immunoassay technique was used, adhering to the manufacturers' protocol. The statistical significance of difference between the two groups was evaluated using the Student's paired t-test.

\section{Result}

The results in this work, table(I) shows that the weight of placenta is an significant and functionally important parameter as it is related to foetal metabolism and villous area. So placental weight, diameter, central thickness and number of cotyledons per placenta $658 \pm 36.5,22.7 \pm 3.44,167.1 \pm 12.22$ and $(20.55 \pm 5.06)$ respectively Increase in diabetic mothers comparison with the normal placenta. In recent study variation was found in umbilical cord insertion between the two groups. Site of umbilical cord insertion was eccentric (37 vs 25), centric (3 vs 10), marginal (0 vs 3 ) velamentous (0 vs 2 ) respectively.Vascular magistral pattern was one case in the GDM group while it was more frequently observed in the normal group. mean birth weight of babies were $3659 \pm 123$. Ratio of newborn weight to placental weight was $5.8 \pm 0.5$ reduced in GDM compared to controls. The result of recent study is sex male/female was $24 / 16$ vs $19 / 21$. In this study the preterm birth only three was in GDM group. Apgar score the mean was $8.9 \pm 0.5$ vs7.3 \pm 0.2 , the mean of Apgar score was less than normal group. (Table 2) was revealed the number of BVs were increased in the GDM group compared to normal was $10.50 \pm 3.33$ vs $19.87 \pm 9.62$, while its mean diameter of blood vessels was $0.04 \pm 0.03$ vs $0.03 \pm 0.02$ found to be significantly decreased. (Table 3) Fasting plasma glucose FBG (mmol/l) 6.30 $\pm 1.02, \beta$-HCG Iu 5001.0 \pm 0.01 , P4 $(\mathrm{ng} / \mathrm{ml}) \quad 72.74 \pm 10.53$, E2 (pmol/L $4501.01 \pm 1334.33$ were increased in the gestational diabetic women. Histological finding Fig. 1(left): The GDM placenta showed increased syncytial knots, thickening of vasculosyncytial membrane, massive fibrinoid necrosis and chorangiosis as fig(1) and Fig.2(right): placental villi showing the Hofbauer cells within the edema and fibrin deposition. 


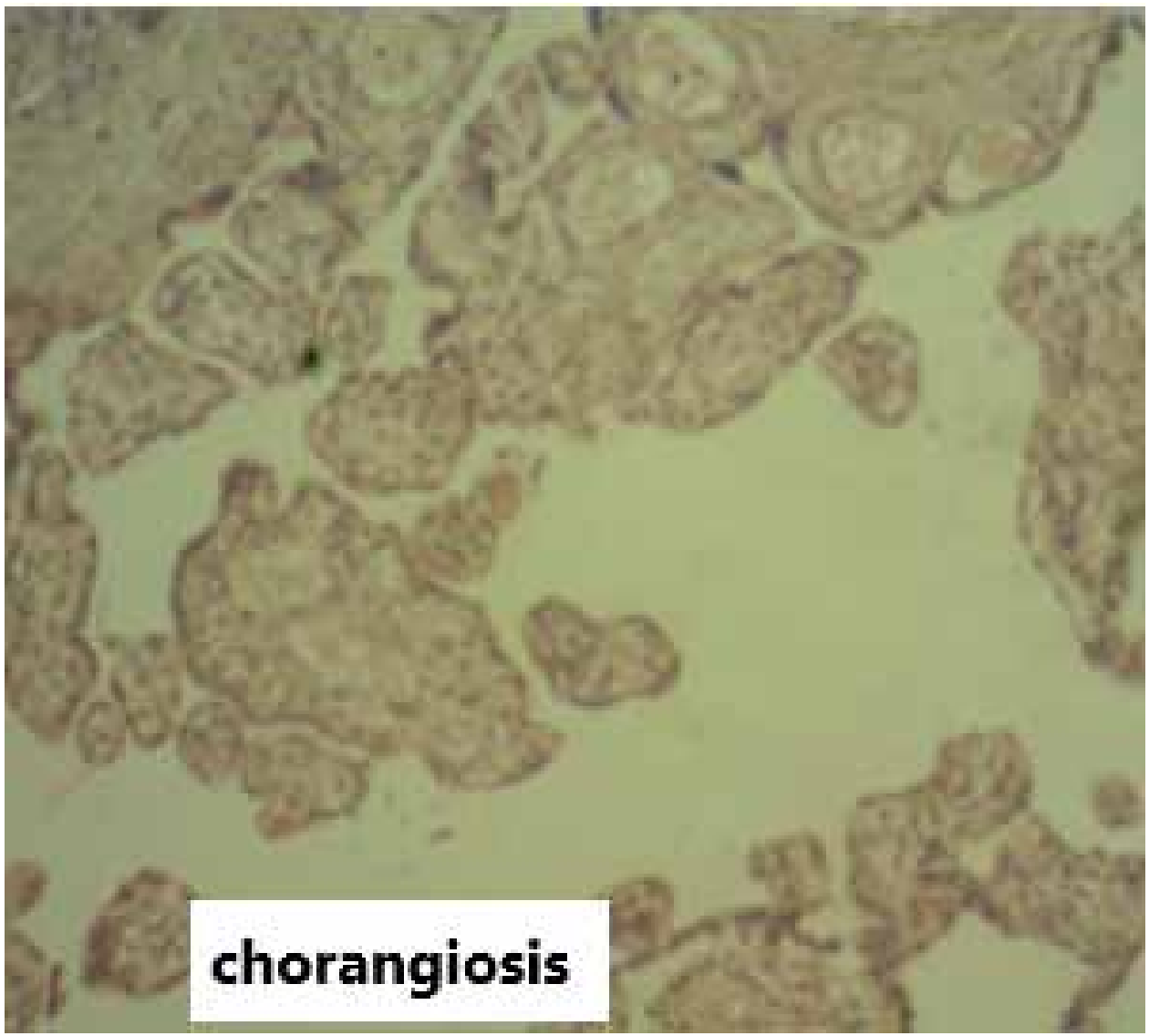

Fig. 1 (Left): The GDM placenta showed chorangiosis with Fetal vessel thrombosis, increased thickening of vasculosyncytial membrane (arrow).

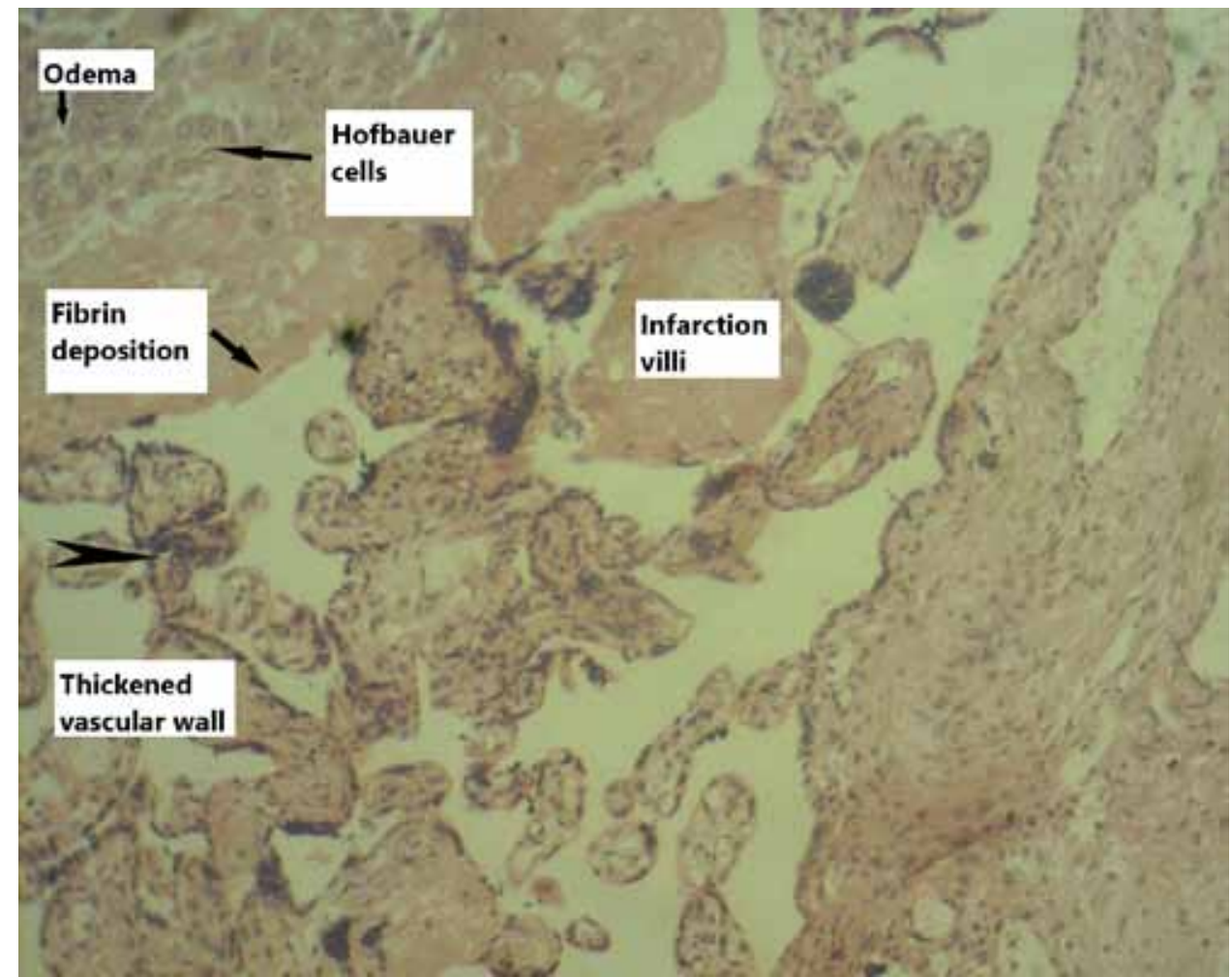

Fig. 2 (Right): Placental villi showing the Hofbauer cells within the edema, fibrin deposition, and infarction villi. 
Table (1): Placenta variables of normal and GDM groups included in the study.

\begin{tabular}{|c|c|c|c|c|}
\hline \multicolumn{2}{|l|}{ Placental variable } & Normal $n=40$ & GDM n=40 & P-value \\
\hline \multicolumn{2}{|l|}{ Placental weight(gm) } & $558 \pm 27.1$ & $658 \pm 36.5$ & $*$ \\
\hline \multicolumn{2}{|l|}{ Central thickness(mm) } & $17.91 \pm 4.67$ & $22.7 \pm 3.44$ & $*$ \\
\hline \multicolumn{2}{|l|}{ Placental diameter (mm) } & $154.5 \pm 6.63$ & $167.1 \pm 12.22$ & $*$ \\
\hline \multirow{4}{*}{ Insertion of umbilical Cord } & Eccentric & 37 & 25 & \\
\hline & Centric & 3 & 10 & \\
\hline & Marginal & 0 & 3 & \\
\hline & Velamentous & 0 & 2 & \\
\hline \multicolumn{2}{|l|}{ No cotyledons per placenta } & $(19.95 \pm 3.42)$ & $(20.55 \pm 5.06)$ & $*$ \\
\hline \multirow{2}{*}{\multicolumn{2}{|c|}{ Vascular pattern }} & Magistral & 10 & 1 \\
\hline & & Dispersal & 30 & 39 \\
\hline \multicolumn{5}{|l|}{ Perinatal outcome } \\
\hline \multicolumn{2}{|l|}{ Birth Weight (g) } & $3608 \pm 104$ & $3659 \pm 123$ & $*$ \\
\hline \multicolumn{2}{|l|}{ Fetoplacental Weight Ratio } & $6.8 \pm 0.3$ & $5.8 \pm 0.5$ & $*$ \\
\hline \multicolumn{2}{|l|}{ Sex male/female } & $24 / 16$ & $19 / 21$ & \\
\hline \multicolumn{2}{|l|}{ Preterm birth } & 0 & 3 & \\
\hline \multicolumn{2}{|l|}{ Apgar score } & $8.8 \pm 0.7$ & $7.8 \pm 0.9$ & $*$ \\
\hline
\end{tabular}

* = significant $\mathrm{p}$-value

Values are presented as mean \pm SD.* significant. TV from GDM placenta showing the increased number of blood vessels (Chorangiosis).

Table (2) Characteristics of Blood Vessels in TV of normal and GDM groups included in the study.

\begin{tabular}{|l|c|c|c|}
\hline Parameter & Control & GDM & p-value \\
\hline Number Blood Vessels in TV/field $\left(\mathrm{mm}^{3}\right)$ & $10.50 \pm 3.33$ & $19.87 \pm 9.62$ & 0.0001 \\
\hline Diameter of Blood Vessels in TV $(\mathrm{mm})$ & $0.04 \pm 0.03$ & $0.03 \pm 0.02$ & 0.0001 \\
\hline
\end{tabular}

Table (3) Comparison variables of hormones in GDM and normal groups.

\begin{tabular}{|l|c|c|c|}
\hline Variables of hormones & GDM & Non GDM & P-value \\
\hline FBG $(\mathrm{mmol} / \mathrm{l})$ & $6.30 \pm 1.02$ & $4.31 \pm 1.02$ & $*$ \\
\hline$\beta-\mathrm{HCG}$ & $5001.0 \pm 0.01$ & $4021 \pm 180.11$ & $*$ \\
\hline P4 $(\mathrm{ng} / \mathrm{ml})$ & $72.74 \pm 10.53$ & $44.83 \pm 14.81$ & $*$ \\
\hline E2 $(\mathrm{pmol} / \mathrm{L})$ & $4501.01 \pm 1334.33$ & $2480.52 \pm 1297.5$ & $*$ \\
\hline
\end{tabular}

$*=p<0.05$ was considered statistically significant

\section{Discussion}

This study was started to determine the comparative between morphological observation of normal and GDM groups included variables as the following table (1).

Site of umbilical cord insertion eccentric and central in most of placentas except in three cases in which there was marginal insertion and two cases velamentous of placenta in GDM group. ${ }^{7}$ Vascular magistral pattern was one case in the GDM group while it was more frequently observed in the normal group. The dispersal pattern showed successive divisions of umbilical vessels with decreasing caliber from center to periphery while the magistral pattern showed fewer divisions of blood vessels 
with noticeably no decrease in diameter from center to periphery. Reduction of uteroplacental circulation results in foetal hypoxia \& intrauterine growth restrected IUGR. ${ }^{8}$ Because macrosomia affects the fetal portion of the placenta, the placental weight, diameter, and central thickness in diabetic mothers increase. The basal lamina of the chorionic capillaries is part of the placental barrier increase in its thickness. ${ }^{3}$ The result of recent study is sex male/female was $24 / 16$ vs $19 / 21$ this observation agreement with study. ${ }^{9}$ Macrosomic newborns are at an increased risk with preterm birth and hypoglycemia. ${ }^{10}$ GDM is associated with an increased risk of additional pregnancy complications, including preterm birth and surgical delivery of the baby is required. ${ }^{11}$ In this study Apgar score the mean was $8.9 \pm 0.5$ vs $7.3 \pm 0.2$, the mean of Apgar score was less than normal group. A study of 94 patients with GDM showed lower Apgar scores and increased incidence of perinatal morbidity of neonates compared to neonates of mothers without impaired glycemic control. ${ }^{12}$ GDM appears to effect the microvascular remodeling at angiogenesis at 3 rd trimester of pregnancy. insulin as a key factor playing a modulatory role in GDM-associated altered angiogenesis. ${ }^{13}$ The angiogenic response to VEGF not only depends on its total concentration, but also on the spatial Vascular magistral pattern was one case in the GDM group while it was more frequently observed in the normal group. The dispersal pattern showed successive divisions of umbilical vessels with decreasing caliber from center to periphery while the magistral pattern showed fewer divisions of blood vessels with noticeably no decrease in diameter from center to periphery. Reduction of uteroplacental circulation results in foetal hypoxia \& intrauterine growth restrected IUGR. ${ }^{8}$ These factors lead to endothelial cell organization within vessels as tip and stalk cell phenotypes ultimately resulting in dynamic developing and branching angiogenesis. ${ }^{14}$

Increased number of capillaries (Chorangiosis), Thickened vessel walls due to endothelial proliferation and thickening of the basement membrane were also identified. Infraction of the villi, fibrin depositions were found in the placenta on histological observations. The decreased elasticity of vessel wall leads to vascular hardening and increased susceptibility to arteriosclerosis, which can lead to a narrowing of the maternal blood vessel lumens, reducing the uteroplacental circulation and thickness of the placental barrier. It has been seen that increased blood glucose levels induce oxidative stress (OS) and subsequent changes of the placental architecture. essentially the vascular properties, which are apparent in GDM. ${ }^{15}$ Placental Hofbauer cells (HBCs) even in low-grade inflammatory states such as GDM. It is concerned in placental vasculogenesis and angiogenesis. This indicates a regulatory, tissue remodeling rather than an inflammatory macrophage phenotype. Also, it was shown that even inflammatory pathologies. HBC are thought to play a role in maternal immunological tolerance against the fetus. ${ }^{16}$ The basal lamina of the chorionic capillaries is part of the placental barrier; therefore, the increase in its thickness will make the placental barrier thicker overall, which can lead to a reduction in the oxygen transport and other nutrients through the barrier. In response to this reduction in pO2, the terminal villi exhibit a hyperplasia that may be partially responsible for the increased placental weight in the diabetic group. ${ }^{3}$ The distance between maternal and fetal circulation is increased because of an increase in the chorionic villi on the surface as well as greater thickness of the syncytiotrophoblast basal membrane due to an increased type IV collagen deposition. The stroma between the villi is edematous, the capillary surface is enlarged due to of vascular neoformation and a greater penetration of these vessels within the villi. Low oxygen partial pressure (pO2) was noticed, which would produce hyperplasia of terminal chorionic villi. In GDM the placenta suffers alterations are correlated to an oxygenation lack in the fetus and variations in the transplacental conveyance of nutrients and other alterations, causing fetal overgrowth by increasing their availability and adverse outcome. ${ }^{17}$ Chorangiosis was defined as the occurrence of 10 or more villi with 10 or more capillaries in 10 or more low power microscopic fields $(\times 10)$. Hydropic villi were diagnosed when large terminal villi were present with edematous fluid and villous macrophages in placenta of GDM. Fetal vessel thrombosis was diagnosed when a large fetal stem villous vessel was partially or completely occluded by a thrombus. Avascular villi were diagnosed when a group of at least five fibrotic avascular villi without inflammation were seen. ${ }^{18}$ These trophoblasts are able to invade and remodel maternal tissues (interstitial EVT) and uterine spiral artery (endovascular EVT), leading to the widening of artery lumen by remodeling normally, reducing the resistance against blood flow that irrigates the fetus. ${ }^{2}$ They recommends that all women undergo a fasting plasma glucose (FPG) test at their first prenatal visit (where a reading $\geq 92 \mathrm{mg} / \mathrm{dL}$ is revealing of GDM), and that women with FPG $<92 \mathrm{mg} / \mathrm{dL}$ undergo a 2-h 75 $\mathrm{g}$ oral glucose tolerance test (OGTT) between 24 and 
28 weeks' gestation. ${ }^{19}$ The hormones produced by EVT contribute to vascular and uterine tissue remodelling and to regulate EVT migration and invasion. ${ }^{1}$ They demonstrated that patients with HCG $>1.04 \mathrm{MoM}$ (Multiple of the Median) and unconjugated E3 (uE3) $\leq$ $0.88 \mathrm{MoM}$ measured in a triple test were associated with GDM development. ${ }^{20}$ Human chorionic gonadotropin $(\mathrm{hCG})$ is a pregnancy-specific hormone that regulates placental development. hCG concentrations vary widely throughout gestation and differ based on fetal sex. Abnormal hCG concentrations are associated with adverse pregnancy outcomes including fetal growth restriction. This was the case for both male and female fetuses. In contrast, high hCG concentrations during the late first trimester were associated with increased fetal growth amongst female, but not male fetuses. Low hCG in the late first trimester is associated with lower birth weight due to a decrease in fetal growth. Fetal sex differences exist in the association of hCG concentrations with fetal growth. ${ }^{21}$ It was reported that the concentrations of the steroids (progesterone and estrogen) are increased in women with GDM. ${ }^{22}$

Financial Disclosure: There is no financial disclosure.

\section{Conflict of Interest: None to declare.}

Ethical Clearance: All experimental protocols were approved under the Kufa College of Science and all experiments were carried out in accordance with approved guidelines.

\section{References}

1. Jia L, Brkićb J, Liua M, Chun F, Yan-Ling W. Placental trophoblast cell differentiation: Physiological regulation and pathological relevance to preeclampsia .Molecular Aspects of Medicine .2013; 34: 981-1023.

2. Mariana A. The endocrine function of human placenta. Reproductive BioMedicine Online.2016; 32: $14-43$.

3. Ashley Moffett, Susan E Hiby, Andrew M Sharkey. The role of the maternal immune system in the regulation of human birthweight.2015; 5; 370(1663).

4. Parham P, Moffett A. Variable NK cell receptors and their MHC class I ligands in immunity, reproduction and human evolution. Nat. Rev. Immunol. 2013; 13: 133-144.
5. ManishaBisht, DC Dhasmana, SS Bist. Angiogenesis: Future of pharmacological modulation Indian J Pharmacol. 2010; 42(1): 2-8 .

6. Standards of medical care in diabetes. American Diabetes Association. 2011; S11-S61.

7. S Pathaka E, Hookb G, Hacketta E, Murdoche N. Cord coiling, umbilical cord insertion and placental shape in an unselected cohort delivering at term: Relationship with common obstetric outcomes. 2010; 31: 963-968.

8. M. YousufSarwar, Nilesh Kumar, NawalKishor Pandey (2013). SERVATIONS ON VASCULAR PATTERN OF CHORIONIC BLOOD VESSELS OF PLACENTAOB. $10 \mathrm{v}(2)$ issue44 Page: 8650865.

9. Ashfaq, M.; Janjua, M. Z. \& Channa, M. A. (2005) Effect of gestational diabetes and maternal hypertension on gross morphology of placenta. J. Ayub. Med. Coll. Abbottabad, 17(1):44-7.

10. TroncosoF, Acurio J, Herlitz K, Aguayo C, Bertoglia P, Guzman-Gutierrez E. Gestational diabetes mellitus is associated with increased promigratory activation of vascular endothelialgrowth factor receptor 2 and reduced expressionof vascular endothelial growth factor receptor 1. PLoS ONE.2017; 12(8).

11. Kc K, Shakya S, Zhang H. Gestational diabetes mellitus and macrosomia: a literature review. Ann NutrMetab. 2015; 2015;66(Suppl 2):14-20 .

12. Jasmine F Plows 1, Joanna L Stanley 2, Philip N Baker 3, Clare M Reynolds 2 and Mark H Vickers() . The Pathophysiology of Gestational Diabetes Mellitus Int. J. Mol. Sci. 2018; 19: 3342.

13. MitrovićM,Stojić S, TešićDS. Theimpactofdiabetes mellitus on the course and outcome of pregnancy during a 5-year follow-up. Military-medical and pharmaceutical review.2014;71(10):907-914.

14. Luis S, Rocío S. Insulin Is a Key Modulator of Fetoplacental Endothelium Metabolic Disturbances in Gestational Diabetes Mellitus. Front Physiol. 2016; 7: 119

15. Eilken HM, Adams RH. Dynamics of endothelial cell behavior in sprouting angiogenesis. Current opinion in cell biology. 2010; 22(5):617-25 .

16. Sankar DK, Bhanu SP, Kiran S. Vasculosyncytial membrane in relation to syncytial knots complicates the placenta in preeclampsia: a histomorphometrical 
study. Anatomy and Cell Biology. 2012;45(2):8691.

17. Schliefsteiner C, Peinhaupt M, Kopp S, Lögl J, Lang-Olip I, Hiden U, Heinemann A, Desoye G and Wadsack C. Human Placental Hofbauer Cells Maintain an Anti-inflammatory M2 Phenotype despite the Presence of Gestational Diabetes Mellitus. Front. Immunol. 2017; 8:888.

18. Carlson B. Embriología Humana y Biologíadel Desarrollo. 5th ed. Ann Arbour, Elsevier. 2014.

19. Debashish B, Santosh K. Histopathological study with immunohistochemical expression of vascular endothelial growth factor in placentas of hyperglycemic and diabetic women. J Lab Physicians. 2017; 9(4): 227-233.
20. Egan A, Vellinga A, Harreiter J. Epidemiology of gestational diabetes mellitus according to IADPSG/ WHO 2013 criteria among obese pregnant women in Europe. Diabetologia.2017; 1-9.

21. Carreno CA, Clifton RG, Hauth JC, Myatt L, Roberts JM, Spong CY. Excessive early gestational weight gain and risk of gestational diabetes mellitus in nulliparous women. Obstet Gynecol. 2012; 119: 1227.

22. MirjanaB, Tim I, Vincent W, Yolanda B.Human chorionic gonadotropin (hCG) concentrations during the late first trimester are associated with fetal growth in a fetal sex-specific manner. Eur $\mathbf{J}$ Epidemio on line. 2016.

23. Kociszewska K. New Insight into Progesteronedependent Signalization. Pharmaceutical Sciences Journal. 2017; 4: 11-22 . 